\title{
Thyroid function in Klinefelter syndrome: a multicentre study from KING group
}

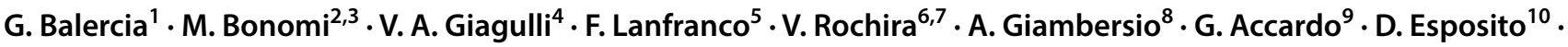 \\ S. Allasia ${ }^{5} \cdot$ B. Cangiano $0^{2,3} \cdot$ S. De Vincentis ${ }^{6,7} \cdot$ R. A. Condorelli ${ }^{11} \cdot$ A. Calogero ${ }^{11}$ • D. Pasquali ${ }^{9}$ on behalf of KING \\ group
}

Received: 19 December 2018 / Accepted: 20 March 2019 / Published online: 25 March 2019

(C) Italian Society of Endocrinology (SIE) 2019

\begin{abstract}
Purpose The prevalence and the etiopathogenesis of thyroid dysfunctions in Klinefelter syndrome (KS) are still unclear. The primary aim of this study was to evaluate the pathogenetic role of hypogonadism in the thyroid disorders described in $\mathrm{KS}$, with the scope to distinguish between patients with KS and hypogonadism due to other causes (Kallmann syndrome, idiopathic hypogonadotropic hypogonadism, iatrogenic hypogonadism and acquired hypogonadotropic hypogonadism after surgical removal of pituitary adenomas) called non-KS. Therefore, we evaluated thyroid function in KS and in non-KS hypogonadal patients.

Methods This is a case-control multicentre study from KING group: Endocrinology clinics in university-affiliated medical centres. One hundred and seventy four KS, and sixty-two non-KS hypogonadal men were enrolled. The primary outcome was the prevalence of thyroid diseases in KS and in non-KS. Changes in hormonal parameters were evaluated. Exclusion criterion was secondary hypothyroidism. Analyses were performed using Student's $t$ test. Mann-Whitney test and Chi-square test.

Results FT4 was significantly lower in KS vs non-KS. KS and non-KS presented similar TSH and testosterone levels. Hashimoto's thyroiditis (HT) was diagnosed in 7\% of KS. Five KS developed hypothyroidism. The ratio FT3/FT4 was similar in both groups. TSH index was 1.9 in KS and 2.3 in non-KS. Adjustment for differences in age, sample size and concomitant disease in multivariate models did not alter the results.

Conclusions We demonstrated in KS no etiopathogenic link to hypogonadism or change in the set point of thyrotrophic control in the altered FT4 production. The prevalence of HT in KS was similar to normal male population, showing absence of increased risk of HT associated with the XXY karyotype.
\end{abstract}

Keywords Klinefelter syndrome $\cdot$ Testosterone $\cdot$ Hypergonadotropic hypogonadism $\cdot$ Thyroid hormones $\cdot$ Thyroid diseases · Hashimoto's thyroiditis

\section{Introduction}

Klinefelter Syndrome (KS) is the most frequent observed sex chromosomal anomaly, with an estimated frequency of 1:500 to 1:1000 men [1]. The classic form of KS, which is

Giancarlo Balercia, Marco Bonomi, Vito A. Giagulli, Fabio Lanfranco and Vincenzo Rochira contributed equally and should be considered co-first authors.

Members of the KING group are listed in Acknowledgements.

D. Pasquali

daniela.pasquali@unicampania.it

Extended author information available on the last page of the article present in the $80-90 \%$ of the cases, is defined by a $47, \mathrm{XXY}$ karyotype, low serum testosterone (T) coupled with elevated gonadotropins, small and firm testes, azoospermia, and tall stature. The clinical presentation of KS syndrome is widely heterogeneous. Only about one in ten males with KS is diagnosed before puberty and $25-50 \%$ in adult age, often during infertility investigation. KS is associated with several clinical conditions coming from both the genetic abnormalities, and hypogonadism. KS patients present several comorbidities including cardiovascular diseases, osteoporosis, diabetes mellitus, and metabolic syndrome, leg ulcers, mostly related to hypogonadism [2-5]. Consequently, KS males need lifelong testosterone replacement treatment. Yet, the therapy does not counteract or prevent all comorbidities. 
It is still unclear whether the extra chromosome $\mathrm{X}$ adds an extra testosterone-independent feature to the complexity of KS comorbidities. Thus, the relationship between clinical phenotype, endocrine dysfunction, KS comorbidities and genetic background needs further investigations [1]. Various thyroid abnormalities were described, mostly in old studies based on few participants, the most common being a low response in serum TSH to a TRH stimulation test $[6,7]$. KS had a general shift toward lower values in distribution of serum FT4 with no compensatory increase in serum TSH [8]. The most likely mechanism suggested is a decrease or change in set point of thyrotrophic control of thyroid function [8]. Recently, a study demonstrated that pubertal KS patients have significantly lower FT3 serum levels than healthy age-matched boys, whereas TSH and FT4 are in the normal reference range [9]. Furthermore, some authors have recently analysed KS comorbidities using data from the Danish National Patient Registry [10]. They searched for differentially expressed genes in peripheral blood and found several expression signatures that correlated with KS status either positively or negatively. Genes involved in 'abnormal thyroid hormone metabolism' were upregulated [10].

To better clarify the prevalence of thyroid diseases and the role of 47XXY as well as the hypogonadism in thyroid dysregulation, we studied thyroid function in 174 patients with KS compared with non-KS hypogonadal men.

\section{Subjects and methods}

\section{Patient population}

We performed a multicentre case-control study, involving the national network of academic or general hospitals named KING (Klinefelter ItaliaN Group). The entire cohort consisted of 174 consecutive KS subjects, and 62 age-matched non-KS hypogonadal men recruited among those regularly attending KING centres since 2015. Inclusion criteria were: (i) a verified KS karyotype (47, XXY) and (ii) a written informed consent. Patients underwent a complete medical and family history evaluation. We examined thyroid function, and thyroid antibodies (Ab). Subjects, who had elevated plasma TPOAb and $\mathrm{TgAb}$ above $350 \mathrm{IU} / \mathrm{ml}$, and thyroid parenchyma heterogeneity with reduced echogenicity, were considered as Hashimoto's thyroiditis (HT) patients. Five KS patients were already under L-thyroxine replacement therapy.

\section{Collection of samples}

Venous blood $(2 \mathrm{~mL})$ from participants was collected by blood banks of the hospitals of the KING centres during the same period of KS and non-KS patients' recruitment.
Samples, blind of identification, treatment or placebo group, were immediately sent for analysis of TSH, FT4, FT3, $\mathrm{TPOAb}$ and $\mathrm{TgAb}$ and total testosterone to the laboratory facility in each hospital institution of the KING groups. The measurements were done on the same day in primary tubes, after blood centrifugation at 3200 RPM for $15 \mathrm{~min}$.

\section{Biochemical data}

As we recruited patients regularly attending KING centres, different methods have been used. In the majority of the cases, serum TSH, FT4, and FT3 concentrations were measured by chemiluminescent immunometric assay (Roche Diagnostics GmbH, Mannheim, Germany). The coefficient of variation was $5.4 \%$ for the FT4 assay. The manufacturer's reference limits are TSH $(0.35-5.5 \mathrm{mIU} / \mathrm{L}), \mathrm{FT} 4$ (10.2-31 pmol/L), and FT3 (3.5-6.5 pmol/L). Detection limit for TSH is $0.005 \mu \mathrm{IU} / \mathrm{mL}$ and functional sensitivity $0.014 \mu \mathrm{IU} / \mathrm{mL}$. Thyroid function alteration was classified according to the American Thyroid Association guidelines $[11,12]$. Clinically overt hyperthyroidism, undetectable to less than $0.1 \mathrm{mIU} / \mathrm{L} \mathrm{TSH}$, more than normal range FT3, and FT4; subclinical hyperthyroidism, undetectable to less than $0.1 \mathrm{mIU} / \mathrm{L}$ TSH, and FT3 and FT4 in the normal range without exogenous T4 intake; clinically overt hypothyroidism, TSH above the upper limit of the normal range $(5 \mathrm{mIU} / \mathrm{L}$ in our assay) and FT4 below $10.2 \mathrm{pmol} / \mathrm{L}$; and subclinical hypothyroidism, TSH above the upper limit of the normal range and FT4 in the normal range $[11,12]$. Total testosterone was measured by immunoassay using a commercial Kit, automated immunoassays (DiaSorin Liaison, Saluggia, VC, Italy).

\section{Exclusion criteria}

KS patients with mosaic forms of chromosomal aneuploidy or any other structural or numerical karyotype anomaly, with AZF microdeletions, were excluded. Moreover, exclusion criterion in non-KS control group was also secondary hypothyroidism defined as undetectable to less than $0.1 \mathrm{mIU} / \mathrm{L}$ TSH, FT4, and FT3 in the lower range without exogenous T4 intake. Subjects with clinically overt thyroid dysfunction, or personal or family history of thyroid disease were excluded from the control sample, which was, therefore, a population of clinically normothyroidal individuals.

\section{Statistical analysis}

Data were tabulated on a spreadsheet and analysed with SPSS statistics 17 software. We calculated mean and standard deviation for continuous variables. 
Analyses were performed using Student's $t$ test. TSH and testosterone were analysed using Mann-Whitney test and, for TPO antibodies Chi-square test analysis was performed.

All analyses were two-sided and $p$ values minor 0.05 were considered statistically significant.

This study was approved by "Comitato Etico Seconda Università degli studi di Napoli- Azienda Ospedaliera Universitaria SUN-AORN Ospedale dei Colli" n 1489, 26.10.2015 to DP.

\section{Results}

\section{Demographic characteristics of the study groups}

The mean age at study entry was $40.6 \pm 1$ (mean \pm SD; range 16-59) and $40.0 \pm 2.4$ (mean $\pm \mathrm{SD}$; range 16-71) years in KS and in non-KS, respectively. The non-KS group was composed by 33 Kallmann syndrome patients, 17 patients with idiopathic hypogonadotropic hypogonadism, 4 patients were affected by iatrogenic hypogonadism, and 8 by acquired hypogonadotropic hypogonadism after surgical removal of pituitary adenomas (Table 1). The comparison between KS and non-KS group allowed evaluating the impact of the hypogonadal condition and the genetic origin of thyroid function alterations in KS. All the subjects were under testosterone replacement treatment. $T$ levels were similar in both KS $(324 \pm 69 \mathrm{ng} / \mathrm{dl})$ and non-KS $(284.7 \pm 36 \mathrm{ng} / \mathrm{dl})$, and in both groups at the lower end of the normal range.

\section{Hashimoto's thyroiditis in KS vs. non-KS patients}

In KS group, $7 \%$ of the patients showed TPOAb positivity, while $4 \%$ out of 62 hypogonadal non-KS group had HT (Table 2). Five KS with HT were diagnosed with hypothyroidism and treated with L-thyroxine substitutive therapy.

Table 1 Diagnoses of patients with hypogonadism not due to Klinefelter syndrome (control group)

\begin{tabular}{lc}
\hline & $\begin{array}{c}\text { Number } \\
\text { of cases }\end{array}$ \\
\hline Kallmann syndrome & 33 \\
After pituitary surgery & 8 \\
Idiopathic & 17 \\
Iatrogenic & 4 \\
Total & 62 \\
\hline
\end{tabular}

Table 2 Clinical, demographical and hormonal data of subjects with Klinefelter syndrome (KS) or with hypogonadism due to other causes (non-KS)

\begin{tabular}{llll}
\hline & KS & non-KS & $p$ \\
\hline $\begin{array}{l}\text { Number of patients } \\
\text { Hashimoto's thyroiditis }\end{array}$ & 174 & 62 & \\
$\quad$ (prevalence) & & $4 \%$ & \\
Age (years) & $40.6 \pm 1.1$ & $40.0 \pm 2.4$ & $\mathrm{~ns}$ \\
Testosterone (ng/dl) & $324.3 \pm 69$ & $284.7 \pm 36.5$ & 0.05 \\
TSH (IU/L) & $1.83 \pm 0.1$ & $2.02 \pm 0.1$ & $\mathrm{~ns}$ \\
FT3 (pmol/L) & $3.3 \pm 0.8$ & $3.2 \pm 0.1$ & $\mathrm{~ns}$ \\
FT4 (pmol/L) & $10.6 \pm 0.3$ & $11.7 \pm 0.4$ & $<0.006$ \\
\hline
\end{tabular}

Data are reported as mean $\pm \mathrm{SD}$

ns not significant

\section{Hypothalamic-pituitary-thyroid axis dysregulation in KS}

Serum FT4 was significantly lower in men with KS versus non-KS [mean (SD): 10.69 (0.2) vs. 11.7 (0.4) pg/ml; $p<0.006$ ] (Table 2; Fig. 1). The FT3/FT4 ratio was similar in KS and in non-KS group (0.32 vs. $0.29, p<0.06)$ (Fig. 2). Nevertheless, KS and non-KS patients showed similar TSH and total T levels [TSH: mean (SD): 1.61 (0.87) vs. 1.75 (1.2) $p=0.1$; testosterone mean (SD): 324 (69) vs. 284 (36) ng/dl, respectively, $p=0.2$ ] (Table 2). We calculated TSH Index, an accurate estimation of the pituitary dysfunction severity in patients with hypopituitarism [14], that was not statistically different between KS compared to non-KS (1.9 vs. 2.1) (Fig. 3). Adjustment for differences in age, sample size and concomitant disease in multivariate models did no alter the results.

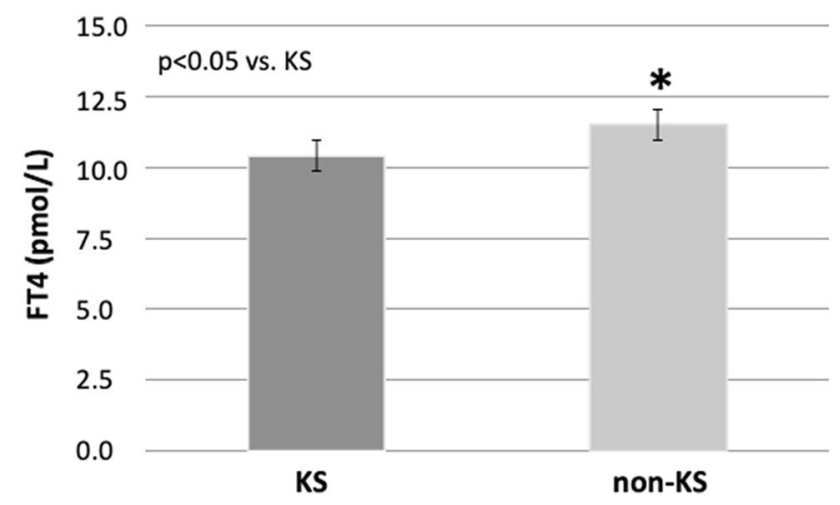

Fig. 1 FT4 levels in Klinefelter syndrome vs. non-Klinefelter syndrome 


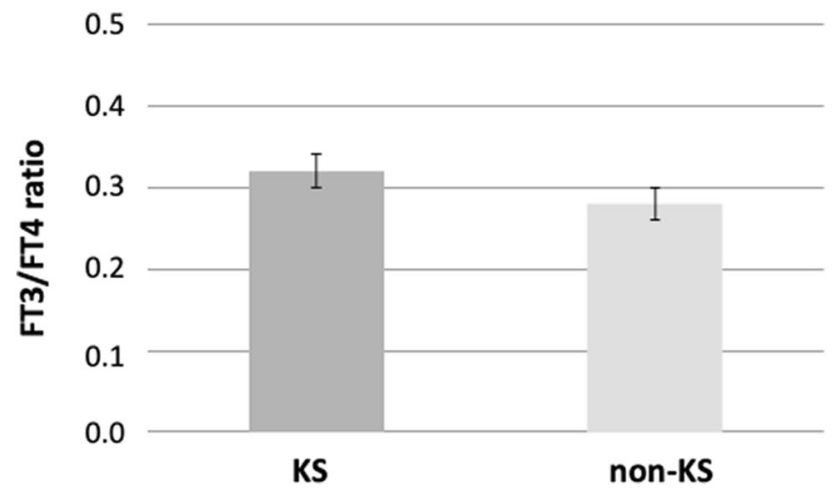

Fig. 2 FT3/FT4 ratio in Klinefelter syndrome vs. non-Klinefelter syndrome. Data are expressed as mean \pm SD

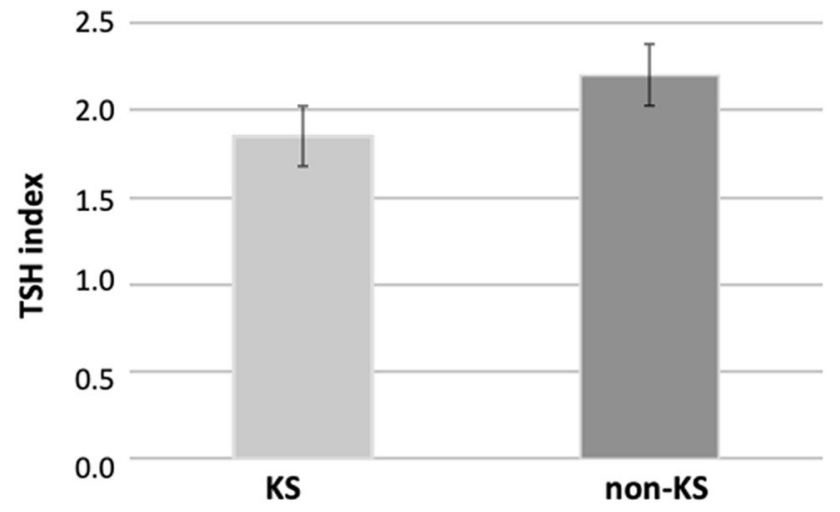

Fig. 3 TSH index in Klinefelter syndrome vs. non-Klinefelter syndrome. Data are expressed as mean $\pm \mathrm{SD}$

\section{Discussion}

The etiopathogenesis of thyroid dysfunction in KS is still under debate. Here, we show lower FT4 in KS, with no changes in FT3 and TSH levels. We have not found a higher prevalence of HT in KS. The cross-talk between thyroid and gonadal hormones is well known. Thyroid hormone regulation of sex steroid synthesis and action in both the brain and gonads are important for gonad development and brain sexual differentiation $[15,16]$. Thyroid transcription factor-1, a homeobox gene that regulates the transcriptional activity of thyroid-specific genes in thyroid follicular cells, occupies a central position within gene networks involved in the hypothalamic control of GnRH production [17]. The effects of thyroid hormones in men and their correlations with sexual behaviour are not yet clear even though several studies show an association between thyroid diseases and altered sexual function (hypoactive sexual desire disorder, premature and delayed ejaculation, and erectile dysfunction) [18]. KS patients have sexual dysfunctions not specifically associated with the syndrome but related to hypogonadism [19] although the ejaculatory control in this category of patients has not yet been studied.

To elucidate if the thyroid dysfunction in KS is related to the peculiarity of $47 \mathrm{XXY}$ condition and/or to T deficiency status, we analysed the occurrence of thyroid diseases in KS and in non-KS hypogonadal men. We have utilized data from a national multicentre study, to overcome the main problem arising from KS studies, the small sample size, that makes difficult to draw strong clinical conclusions. We confirm that males with KS have a shift toward lower values in distribution of serum FT4 [8]. In particular, we have found a significant reduction of FT4 level in KS compared to non-KS, with no statistical differences in TSH and FT3 levels. To verify the hypothesis of an altered set point of thyrotrophic control of thyroid function in KS [8], we have performed TSH index calculation. TSH index is an "FT4-adjusted TSH" that corrects for any physiological TSH suppression, to provide a true estimate of pituitary thyrotrophic function and any pathological pituitary suppression [14]. TSH index was not statistically different in KS and non-KS, suggesting an adequate hypothalamic-pituitary-thyroid axis control. We could speculate that, since in healthy and iodine-sufficient individuals, the majority of thyroid hormone produced is T4, synthesized exclusively by the thyroid gland, with a smaller amount ( $\sim 20 \%)$ of T3, which is produced by the thyroid and in peripheral tissues via deiodination of circulating T4, KS patients could have an altered T4 production by the thyroid gland. The novel approach with the comparison of FT4 levels between KS and non-KS patients give us the opportunity to clarify that $\mathrm{T}$ deficit, independently of when it started, cannot be considered as a causal determinant in the pathophysiology of this condition. Metabolic syndrome and insulin resistance are specific features of KS [1-3, 20-22] and FT3/FT4 ratio has a better predictive power for metabolic syndrome than TSH [23]. We found comparable FT3/FT4 ratio both in KS and in non-KS, suggesting a similar predisposition to increased risk of metabolic syndrome parameters and insulin resistance. Then, we took the opportunity to study the prevalence of Hashimoto's thyroiditis (HT) among KS that resulted to be $7.0 \%$. The prevalence of HT in KS was similar to that reported in healthy male volunteers in the Pescopagano survey [24, 25]. In fact, in this comprehensive study of thyroid disease in Italy $17.3 \%$ of women and $7.0 \%$ of male were affected by HT $[24,25]$. The X chromosome contains the largest number of immune-related genes of the whole human genome [26]. Skewed X chromosome inactivation has been considered one of the key factors that influence the female predisposition to autoimmunity [27]. Laboratory studies have shown high levels 
of autoantibodies to the thyroid gland among people with KS [28]. An English national cohort study of people with KS found significantly increased risks of several autoimmune diseases, including acquired hypothyroidism [29]. The extra X chromosome could predispose KS to higher prevalence of HT. Our Italian national cohort results do not support this hypothesis and are in contrast with previous studies, in particular considering the prevalence of HT in our KS cohort. This could be explained by the limits of a dataset from hospital discharge records and/or the diagnostic criteria of HT [28, 29]. Furthermore, a recent study based on data from the Danish National Patient Registry [10] analysed KS comorbidities, searching for differentially expressed genes in peripheral blood. There were genes causative of several Online Mendelian Inheritance in Man (OMIM) diseases such as 'abnormal thyroid hormone metabolism', 'epilepsy' and 'deafness'. These data indicate that more factors are involved in thyroid dysregulation in KS other than increased risk of autoimmune diseases. In previous studies, the most likely mechanism of thyroid abnormalities found in KS is an alteration in set point of thyrotrophic regulation of thyroid function [6-9]. It is quite difficult to establish what are the causes and the effects of thyroid dysfunction in KS. However, we can exclude the influence of hypogonadism leading to a dysregulation of hypothalamic-pituitary-thyroid axis.

In conclusion, our study indicates that KS presents an impaired production of T4 by the thyroid gland, suggesting a different set point of the thyroid axis versus patients with hypogonadism due to other causes. No evidences of etiopathogenetic link to the hypogonadal status or change in set point of thyrotrophic control were found. Further studies are needed to better understand the underlying mechanism(s) of thyroid dysregulation that may include evaluation of deiodinase activity, iodine intake and urinary iodine excretion.

Acknowledgements This study was performed on behalf of the Klinefelter ItaliaN Group (KING). Coordinators: Antonio Aversa (Catanzaro), Giancarlo Balercia (Ancona), Marco Bonomi (Milan), Aldo Calogero (Catania), Giovanni Corona (Bologna), Francesco Giorgino (Bari), Andrea Fabbri (Rome), Alberto Ferlin (Padua), Emanuele Ferrante (Milan), Felice Francavilla (L'Aquila), Vito Giagulli (Conversano, Bari), Emmanuele Jannini (Rome), Fabio Lanfranco (Turin), Mario Maggi (Florence), Daniela Pasquali (Naples), Rosario Pivonello (Naples), Alessandro Pizzocaro (Milan), Antonio Radicioni (Rome), Vincenzo Rochira (Modena), Linda Vignozzi (Florence). Members: Marco Barchi (Rome), Biagio Cangiano (Milan), Rosita A. Condorelli (Catania), Giuliana Cordeschi (L'Aquila), Settimio D'Andrea (L'Aquila), Antonella Di Mambro (Padua), Carlo Foresta (Padua), Sandro Francavilla (L'Aquila), Andrea Garolla (Padua), Lara Giovannini (Ancona), Antonio R. M. Granata (Modena), Sandro La Vignera (Catania), Giovanna Motta (Turin), Luciano Negri (Milan), Fiore Pelliccione (Milan), Luca Persani (Milan), Ciro Salzano (Naples), Daniele Santi (Modena), Riccardo Selice (Padua), Manuela Simoni (Modena), Carla Tatone (L'Aquila), Giacomo Tirabassi (Ancona), Alberto Stefano Tresoldi (Milan) and Enzo Vicari (Catania). The KING belongs to the Italian Society of Andrology and Sexual Medicine (SIAMS) and aims to promote all the activities, clinical, research, and informative, concerning KS in Italy.

Funding This research did not receive any specific grant from any funding agency in the public, commercial or not-for-profit sector.

\section{Compliance with ethical standards}

Conflict of interest The authors have nothing to disclose.

\section{References}

1. Bonomi M, Rochira V, Pasquali D, Balercia G, Jannini EA, Ferlin A; Klinefelter ItaliaN Group (KING) (2017) Klinefelter syndrome (KS): genetics, clinical phenotype and hypogonadism. J Endocrinol Invest 40:123-134

2. Kanakis GA, Nieschlag E (2018) Klinefelter syndrome: more than hypogonadism. Metabolism 286:135-144

3. Calogero AE, Giagulli VA, Mongiò LM, Triggiani V, Radicioni AF, Jannini EA, Pasquali D; Klinefelter ItaliaN Group (KING) (2017) Klinefelter syndrome: cardiovascular abnormalities and metabolic disorders. J Endocrinol Invest 40:705-712

4. Salzano A, Arcopinto M, Marra AM et al (2016) Klinefelter syndrome, cardiovascular system, and thromboembolic disease: review of literature and clinical perspectives. Eur J Endocrinol 175:R27-40

5. Di Minno MN, Esposito D, Di Minno A et al (2015) Increased platelet reactivity in Klinefelter men: something new to consider. Andrology 3:876-878

6. Smals AG, Kloppenborg PW, Lequin RL, Beex L, Ross A, Benraad TJ (1977) The pituitary-thyroid axis in Klinefelter's syndrome. Acta Endocrinol (Copenh) 84:72-79

7. Wortsman J, Moses HW, Dufau ML (1986) Increased incidence of thyroid disease among men with hypergonadotropic hypogonadism. Am J Med 80:1055-1059

8. Bjørn AM, Bojesen A, Gravholt CH, Laurberg PJ (2009) Hypothyroidism secondary to hypothalamic-pituitary dysfunction may be part of the phenotype in Klinefelter syndrome: a case-control study. J Clin Endocrinol Metab 94:2478-2481

9. Tahani N, Ruga G, Granato S et al (2017) A combined form of hypothyroidism in pubertal patients with non-mosaic Klinefelter syndrome. Endocrine 55:513-518

10. Belling K, Russo F, Jensen AB et al (2017) Klinefelter syndrome comorbidities linked to increased $\mathrm{X}$ chromosome gene dosage and altered protein interactome activity. Hum Mol Gen 26:1219-1229

11. Jonklaas J, Bianco AC, Bauer AJ et al (2014) American Thyroid Association Task Force on Thyroid Hormone Replacement. Guidelines for the treatment of hypothyroidism: prepared by the American Thyroid Association task force on thyroid hormone replacement. Thyroid 24:1670-1751

12. Ross DS, Burch HB, Cooper DS et al (2016) American Thyroid Association Guidelines for diagnosis and management of hyperthyroidism and other causes of thyrotoxicosis. Thyroid 26:1343-1421

14. Jostel A, Ryder WD, Shalet SM (2009) The use of thyroid function tests in the diagnosis of hypopituitarism: definition and evaluation of the TSH Index. Clin Endocrinol (Oxf) 71:529-534

15. Duarte-Guterman P, Navarro-Martín L, Trudeau VL (2014) Mechanisms of crosstalk between endocrine systems: regulation of sex steroid hormone synthesis and action by thyroid hormones. Gen Comp Endocrinol 203:69-85 
16. Wayne Meikle A (2004) The Interrelationships Between Thyroid Dysfunction and Hypogonadism in Men and Boys. Thyroid 14(Suppl 1):S17-25

17. De Felice M, Di Lauro R (2004) Thyroid development and its disorders: genetics and molecular mechanisms. Endocr Rev 25:722-746

18. Carosa E, Lenzi A, Jannini EA (2018) Thyroid hormone receptors and ligands, tissue distribution and sexual behaviour. Mol Cell Endocrinol 467:49-59

19. Vignozzi L, Corona G, Forti G, Jannini EA, Maggi M (2010) Clinical and therapeutic aspects of Klinefelter's syndrome: sexual function. Mol Hum Reprod 16:418-424

20. Pasquali D, Arcopinto M, Renzullo A et al (2013) Cardiovascular abnormalities in Klinefelter syndrome. Int J Cardiol 168:754-759

21. Groth KA, Skakkebæk A, Høst C, Gravholt CH, Bojesen AJ (2013) Clinical review: Klinefelter syndrome-a clinical update. J Clin Endocrinol Metab 98:20-30

22. Salzano A, D'Assante R, Heaney LM et al (2018) Klinefelter syndrome, insulin resistance, metabolic syndrome, and diabetes: review of literature and clinical perspectives. Endocrine 61:194-203

23. Park SY, Park SE, Jung SW, Jin HS, Park IB, Ahn SV, Lee S (2017) Free triiodothyronine/free thyroxine ratio rather than thyrotropin is more associated with metabolic parameters in healthy euthyroid adult subjects. Clin Endocrinol (Oxf) 87:87-96

24. Aghini-Lombardi F, Antonangeli L, Martino E et al (1999) J Clin Endocrinol Metab 84:561-566
25. McLeod DS, Cooper DS (2012) The incidence and prevalence of thyroid autoimmunity. Endocrine 42:252-265

26. Zaletel K, Gaberšček S (2011) Hashimoto's Thyroiditis: From Genes to the Disease. Curr Genomics 12:576-588

27. Brix TH, Knudsen GP, Kristiansen M, Kyvik KO, Orstavik KH, Hegedüs L (2005) High frequency of skewed X-chromosome inactivation in females with autoimmune thyroid disease: a possible explanation for the female predisposition to thyroid autoimmunity. J Clin Endocrinol Metab 90:5949-5953

28. Vallotton M, Forbes A (1967) Autoimmunity in gonadal dysgenesis and Klinefelter's syndrome. Lancet 289:648-651

29. Seminog OO, Seminog AB, Yeates D, Goldacre MJ (2015) Associations between Klinefelter's syndrome and autoimmune diseases: English national record linkage studies. Autoimmunity 48:125-128

13. Aghini-Lombardi F, Antonangeli L, Martino E et al (1999) The spectrum of thyroid disorders in an iodine-deficient community: the Pescopagano survey. J Clin Endocrinol Metab 84:561-566

Publisher's Note Springer Nature remains neutral with regard to jurisdictional claims in published maps and institutional affiliations.

\section{Affiliations}

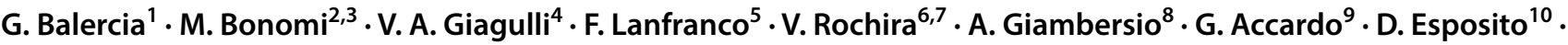 S. Allasia ${ }^{5} \cdot$ B. Cangiano ${ }^{2,3} \cdot$ S. De Vincentis ${ }^{6,7} \cdot$ R. A. Condorelli ${ }^{11}$ - A. Calogero ${ }^{11}$ - D. Pasquali ${ }^{9}$ on behalf of KING gro up}

1 Division of Endocrinology, Department of Clinical and Molecular Sciences, Umberto I Hospital, Polytechnic University of Marche, Ancona, Italy

2 Department of Clinical Sciences and Community Health, University of Milan, Milan, Italy

3 Division of Endocrine and Metabolic Diseases, Lab of Endocrine and Metabolic Research, IRCCS Istituto Auxologico Italiano, Milano, Italy

4 Outpatient Clinic for Endocrinology and Metabolic Diseases, Conversano Hospital, Conversano, Bari, Italy

5 Division of Endocrinology, Diabetology, and Metabolism, Department of Medical Sciences, University of Turin, Turin, Italy

6 Unit of Endocrinology, Department of Biomedical, Metabolic and Neural Sciences, University of Modena and Reggio Emilia, Modena, Italy
7 Unit of Endocrinology, Department of Medical Specialties, Azienda Ospedaliero-Universitaria Di Modena, Ospedale Civile Di Baggiovara, Modena, Italy

8 Healt Center "Maria Teresa Di Calcutta, Potenza, Italy

9 Department of Advanced Medical And Surgical Sciences, University of Campania “Luigi Vanvitelli”, 80138 Naples, Italy

10 Department of Internal Medicine and Clinical Nutrition, Institute of Medicine, Sahlgrenska Academy, University of Gothenburg, Gothenburg, Sweden

11 Department of Clinical and Experimental Medicine, University of Catania, Policlinico "G. Rodolico", Catania, Italy 